University of Wollongong

Research Online

Faculty of Engineering - Papers (Archive)

Faculty of Engineering and Information

Sciences

May 1993

\title{
High-field Zeeman effect of shallow acceptors in germanium
}

P. Fisher

University of Wollongong, pfisher@uow.edu.au

G. J. Takacs

University of Wollongong, gjt@uow.edu.au

R. Vickers

University of Wollongong, rv@uow.edu.au

A. D. Warner

University of Wollongong

Follow this and additional works at: https://ro.uow.edu.au/engpapers

Part of the Engineering Commons

https://ro.uow.edu.au/engpapers/270

\section{Recommended Citation}

Fisher, P.; Takacs, G. J.; Vickers, R.; and Warner, A. D.: High-field Zeeman effect of shallow acceptors in germanium 1993.

https://ro.uow.edu.au/engpapers/270

Research Online is the open access institutional repository for the University of Wollongong. For further information contact the UOW Library: research-pubs@uow.edu.au 


\title{
High-field Zeeman effect of shallow acceptors in germanium
}

\author{
P. Fisher, G. J. Takacs, R. E. M. Vickers, and A. D. Warner \\ Department of Physics, University of Wollongong, Wollongong, New South Wales 2522, Australia
}

(Received 4 January 1993)

\begin{abstract}
Zeeman absorption spectra have been obtained for $B$ and $\mathrm{Ga}$ in $\mathrm{Ge}$ for $\mathbf{B} \|\langle 100\rangle$ in the Voigt configuration with plane-polarized radiation. All twelve allowed transitions were observed for both the $G$ and $D$ lines. The corresponding excited states of these two lines for both impurities behave identically; two recent theoretical results are in good agreement. The measurements are a sensitive probe of the ground states; there are differences between the behavior of these for the two acceptors.
\end{abstract}

A fundamental property remaining to be fully understood for impurities in elemental bulk semiconductors is the detailed nature of their ground states. No successful $a b$ initio calculation has been performed which predicts the energies of these states and their variation from one chemical species to another. This deficiency is partly due to the lack of definitive experimental data on the details of these states. One very effective and direct way by which experimental information can be obtained about a quantum state is to split it into its separate Zeeman substates and study the optical spectra associated with these. The present paper gives the results of a detailed highresolution Zeeman study at high magnetic fields $\mathbf{B}(B \leq 7$ $T)$ of the optical absorption line spectra of two of the classic shallow acceptors, B (Ref. 1) and Ga, in Ge, the classical and archetypal semiconductor. The observations have been carried out in the Voigt configuration for $\mathbf{B} \|\langle 100\rangle$ using plane-polarized radiation. All twelve allowed Zeeman components of the $D$ and $G$ lines $^{2}$ are observed thus giving complete and precise information about not only the excited states but the ground states. Small but significant differences in the behavior of the latter for the two impurities are observed. The results are compared with two recent, detailed numerical calculations of this effect. ${ }^{3,4}$

This is not the first study of the Zeeman effect of group-III impurities in Ge. Over the past twenty years, several such investigations have been carried out. ${ }^{5-9}$ In all but the most recent, ${ }^{9}$ the field strength was limited to a maximum of $2.5 \mathrm{~T}$. Both absorption and photoconductive measurements were made, most of which utilized the Faraday configuration. The recent study ${ }^{9}$ extended the photoconductive data to $5.6 \mathrm{~T}$ in the Faraday arrangement but did not observe all the components of the various lines examined. In addition, interpretation relied on either somewhat inaccurate theoretical results ${ }^{10}$ or those in which interactions between states are omitted, ${ }^{11}$ although a precursor of the treatment of Refs. 3 and 4 was given in Ref. 7. None of the above experimental results permitted the field dependence of the ground-state splitting to be determined with precision except to obtain $g$ factors which, as will be seen, only have significance for most of the states at very small magnetic fields.

The main calculations to which reference will be made use the classification introduced in Ref. 12 for describing the acceptor energy states and thus, here, these will be la- beled according to this notation. In Ref. 12, the symmetry of the zero-field Hamiltonian is taken as $\bar{O}_{h}$; this reduces to $\bar{C}_{4 h}$ for $\mathbf{B} \|\langle 100\rangle$. Under this reduction, the "even-parity" and the "odd-parity" states, $n \Gamma_{8}^{+}\left(\bar{O}_{h}\right)$ and $n \Gamma_{8}^{-}\left(\bar{O}_{h}\right)$, respectively, become $\sum_{i} n \Gamma_{i}^{+}\left(\bar{C}_{4 h}\right)$ and $\sum_{f} n \Gamma_{f}^{-}\left(\bar{C}_{4 h}\right)$, respectively, where $i, f=5,6,7,8$ and $n=1,2,3$, etc. The electric dipole selection rules for this direction of B for "even-parity" to "odd-parity" transitions are given by $i \rightarrow f$, where $i=f$ for $\mathbf{E} \| \mathbf{B}\left(\mathbf{E}_{\|}\right)$, $|i-f|=1,3$ for $\mathbf{E} \perp \mathbf{B}\left(\mathbf{E}_{1}\right)$; here $i$ and $f$ also refer to the initial and final states, respectively, and $\mathbf{E}$ is the electric field of the radiation. Zeeman components are labeled as $X(i, f)$, where $X=G, E, D$, etc.; for example, the transition $1 \Gamma_{5}^{+}\left(\bar{C}_{4 h}\right) \rightarrow 1 \Gamma_{6}^{-}\left(\bar{C}_{4 h}\right)$ is labeled as $G(5,6)$ and is only allowed for $\mathbf{E}_{\perp}$, it being known that $1 \Gamma_{8}^{-}\left(\bar{O}_{h}\right)$ is the unperturbed final state of the $G$ line. ${ }^{2}$ It should be noted that the subscripts on the symmetry labels in the notation of Ref. 12 are not the same as those of Ref. 11 for the even-parity states, whereas the magnetic quantum numbers, $m_{j}=\frac{3}{2}, \frac{1}{2},-\frac{1}{2},-\frac{3}{2}$, are the same. A different correlation holds for the odd-parity states; Table I in Ref. 3 reconciles the two notations.

The absorption spectra have been obtained using a Fourier transform spectrometer interfaced to a 7-T splitcoil superconducting magnet; the radiation was detected with a silicon bolometer operating at $\sim 1.5 \mathrm{~K}$. The Zeeman spectrum of the $G$ and $D$ lines of $\mathrm{Ga}$ impurity for a field of $7 \mathrm{~T}$ and sample temperature of $4.2 \mathrm{~K}$ is given in Fig. 1 for $\mathbf{B} \|\langle 100\rangle$; the unapodized resolution is 0.037 $\mathrm{cm}^{-1}$. The results obtained for $\mathbf{E}_{\|}$are represented by the dashed spectrum while those for $\mathbf{E}_{\perp}$ are given by the full curve; an almost identical spectrum is obtained for $B$ in Ge. As is known, for $B \lesssim 3 \mathrm{~T}$, at moderate resolution, the $D$ line yields four components for both $\mathbf{E}_{\|}$and $\mathbf{E}_{\perp}$ (Ref. 5) (also see Refs. 6, 7, and 9). As can be seen in Fig. 1, the present observations reveal an additional four components for $\mathbf{E}_{\perp}$; similarly for the $G$ line. This is so for both impurities and comparison of their individual spectra, at low and high fields, shows the evolution of the components; preliminary results for the $G$ line of B have been presented elsewhere. ${ }^{1}$ Not all components are observed at all fields; thus, for example, at $B=7 \mathrm{~T}$, the components labeled $G(6,6)$ and $D(5,8)$ are too weak to be detected. In Fig. 1, the energies at which these should occur are indicated by dotted arrows and have been de- 


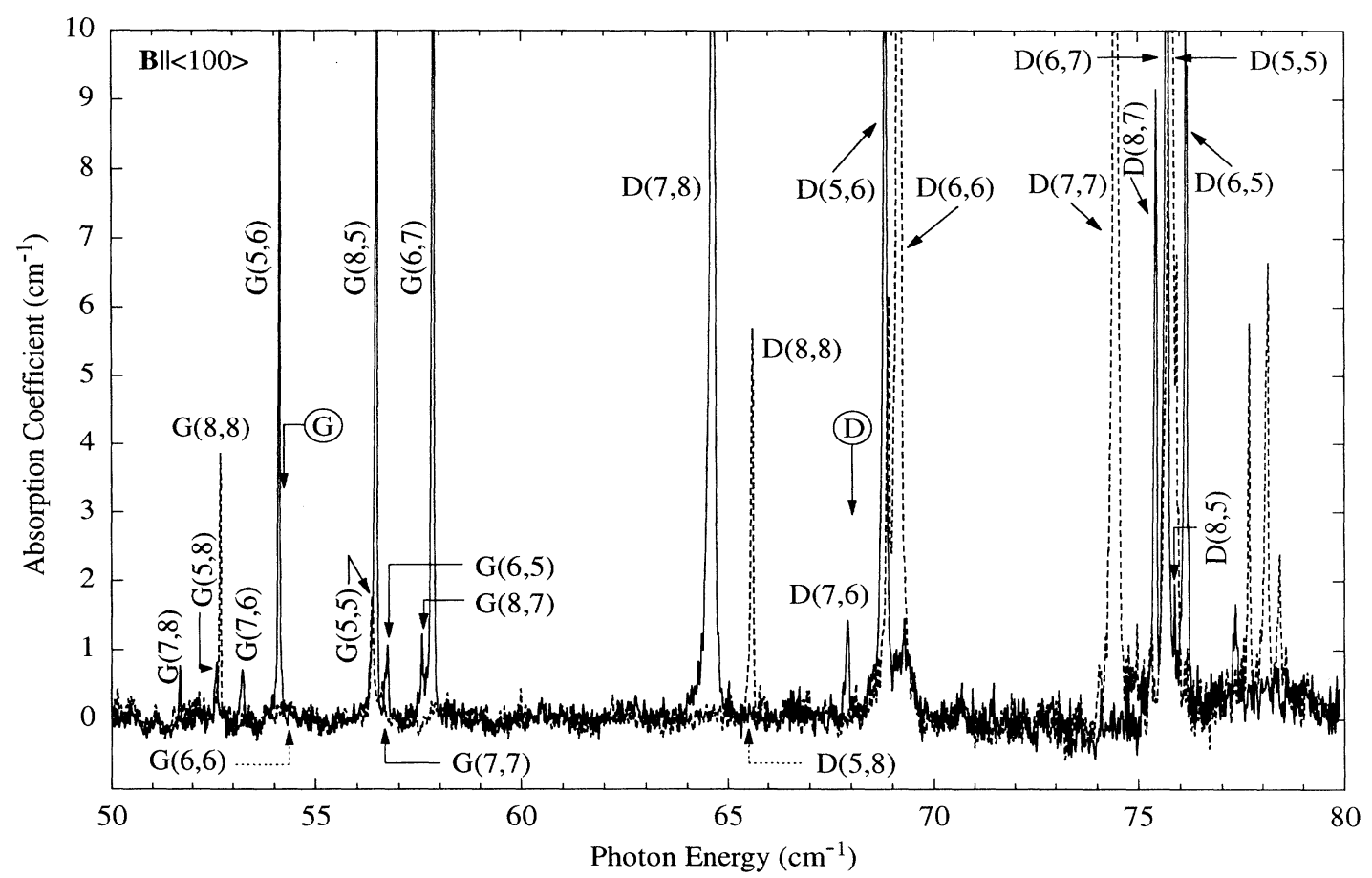

FIG. 1. Zeeman spectrum of the $G$ and $D$ lines of $\mathrm{Ga}$ in $\mathrm{Ge}$ for $B=7 \mathrm{~T}$. The full curves are for $\mathbf{E} \perp \mathbf{B}$ and the dashed curves for $\mathbf{E} \| \mathbf{B}$; temperature is $4.2 \mathrm{~K}$. The encircled letters and associated arrows indicate the energies of the unperturbed lines.

duced from the fits to their energies at lower fields. Also, several features are not labeled; those beyond $77 \mathrm{~cm}^{-1}$ arise from the splitting of the $C$ line, while those interwoven with the $D$ components have their origin in the $E$ line.

The theoretical treatments ${ }^{3,4}$ agree regarding the ordering of the Zeeman sublevels of the $1 \Gamma_{8}^{-}\left(\bar{O}_{h}\right)$ and $2 \Gamma_{8}^{-}\left(\bar{O}_{h}\right)$ states, the unperturbed excited states of the $G$ and $D$ lines, respectively; ${ }^{2}$ one calculation extends to $5 \mathrm{~T}$ (Ref. 3) while the other to $\sim 10 \mathrm{~T}^{4}$ Thus, here, it is assumed that the Zeeman substates of these excited states are ordered as predicted., ${ }^{3,4}$ With this assumption and knowing that the ground-state splitting is very small at low fields, ${ }^{7,8}$ a detailed argument (not given here) which invokes the energies of the very sharp $G$ components and the selection rules leads to only one possible configuration of the Zeeman sublevels of the ground state $1 \Gamma_{8}^{+}\left(\bar{O}_{h}\right)$ for each impurity. Once the behavior of the ground state as a function of field has been established, the energies of the $D$ components for $\mathbf{E}_{\|}$, together with the selection rules, enable the splitting of $2 \Gamma_{8}^{-}\left(\bar{O}_{h}\right)$ to be determined. With the energies of the Zeeman sublevels of both this and the ground state known, the results are verified by comparing the energies of all eight $D$ components observed for $\mathbf{E}_{1}$ with those given by the experimental energy level scheme; excellent agreement is obtained for both impurities.

It is found that the experimental behavior of $1 \Gamma_{8}^{-}\left(\bar{O}_{h}\right)$ and $2 \Gamma_{8}^{-}\left(\bar{O}_{h}\right)$ for both $\mathrm{B}$ and $\mathrm{Ga}$ are identical and close to that calculated. For example, Fig. 2 shows the splitting of $2 \Gamma_{8}^{-}\left(\bar{O}_{h}\right)$ for B and $\mathrm{Ga}$ as obtained experimentally and theoretically. The experimental results are shown as data points, while the full curves ${ }^{3}$ and the dashed curves ${ }^{4}$ give the theoretical behavior. In constructing this figure, the experimental behavior of $2 \Gamma_{8}^{-}\left(\bar{C}_{4 h}\right)$ has been taken to be that predicted in Ref 4; these theoretical results have been used since they embrace the range of the experimen-

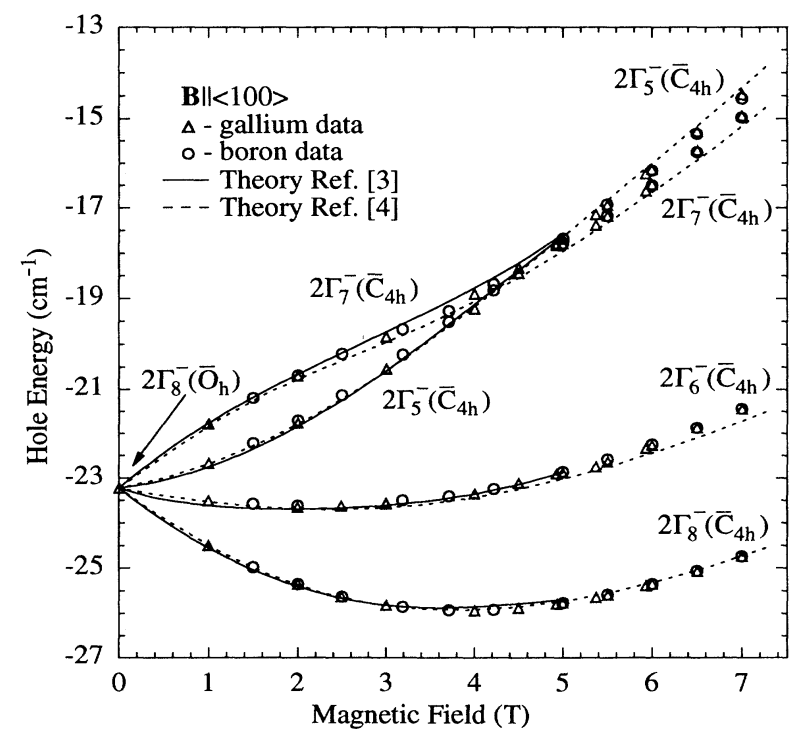

FIG. 2. Experimental Zeeman splitting of $2 \Gamma_{8}^{-}\left(\bar{O}_{h}\right)$, the excited state of the $D$ line, of $\mathrm{B}$ and $\mathrm{Ga}$ in $\mathrm{Ge}$. The theoretical behavior, Refs. 3 and 4, is also shown. 
tal data. The excellent agreement between theory and experiment of the somewhat complex behavior of $2 \Gamma_{8}^{-}\left(\bar{O}_{h}\right)$, in particular $2 \Gamma_{7}^{-}\left(\bar{C}_{4 h}\right)$, is a good indication of the ability of the theory to describe the excited states of these impurities. For the splitting of the $1 \Gamma_{8}^{-}\left(\bar{O}_{h}\right)$ states, both calculations $^{3,4}$ give essentially identical results over their common range of field, but the agreement with experiment is not as satisfactory as that for $2 \Gamma_{8}^{-}\left(\bar{O}_{h}\right)$.

The experimental behavior of the ground state $1 \Gamma_{8}^{+}\left(\bar{O}_{h}\right)$ of $\mathrm{B}$, as determined from the energies of the very sharp $G$ components, is illustrated by the dashed curves fitted through the data points in Fig. 3. Also shown is the predicted behavior of this state as determined in Ref. 3 (full curves). Since it is not possible experimentally to find the shift of the center of gravity of each of the two manifolds involved in a given transition, an artifice has been used to construct Fig. 3. This is based on the experimental observation, supported by both theoretical treatments, ${ }^{3,4}$ that the energy spacing of the Zeeman states, $1 \Gamma_{5}^{+}\left(\bar{C}_{4 h}\right)\left(+\frac{1}{2}\right)$ and $1 \Gamma_{6}^{+}\left(\bar{C}_{4 h}\right)\left(-\frac{1}{2}\right)$, varies almost linearly with field; here the values of $m_{j}$ for these states are given in the second parentheses. In Fig. 3 , for both B and theory, the center of gravity of these two states is held constant with field and taken to be the zero of energy. Thus, the above two states are symmetrically disposed about the field axis and vary linearly. The field dependences of the other two Zeeman substates of the ground state, $1 \Gamma_{7}^{+}\left(\bar{C}_{4 h}\right)\left(-\frac{3}{2}\right)$ and $1 \Gamma_{8}^{+}\left(\bar{C}_{4 h}\right)\left(+\frac{3}{2}\right)$, have been obtained by adding to the energy of $1 \Gamma_{6}^{+}\left(\bar{C}_{4 h}\right)\left(-\frac{1}{2}\right)$ the energy differences of $1 \Gamma_{6}^{+}\left(\bar{C}_{4 h}\right)\left(-\frac{1}{2}\right)$ and $1 \Gamma_{7}^{+}\left(\bar{C}_{4 h}\right)\left(-\frac{3}{2}\right)$, and $1 \Gamma_{6}^{+}\left(\bar{C}_{4 h}\right)\left(-\frac{1}{2}\right)$ and $1 \Gamma_{8}^{+}\left(\bar{C}_{4 h}\right)\left(+\frac{3}{2}\right)$, respectively. These differences are obtained directly from the energies of the appropriate pairs of Zeeman components. In Fig. 3 it is seen that the experimental splitting of the $\pm \frac{1}{2}$ substates is significantly different from the theoretical prediction. In contrast,

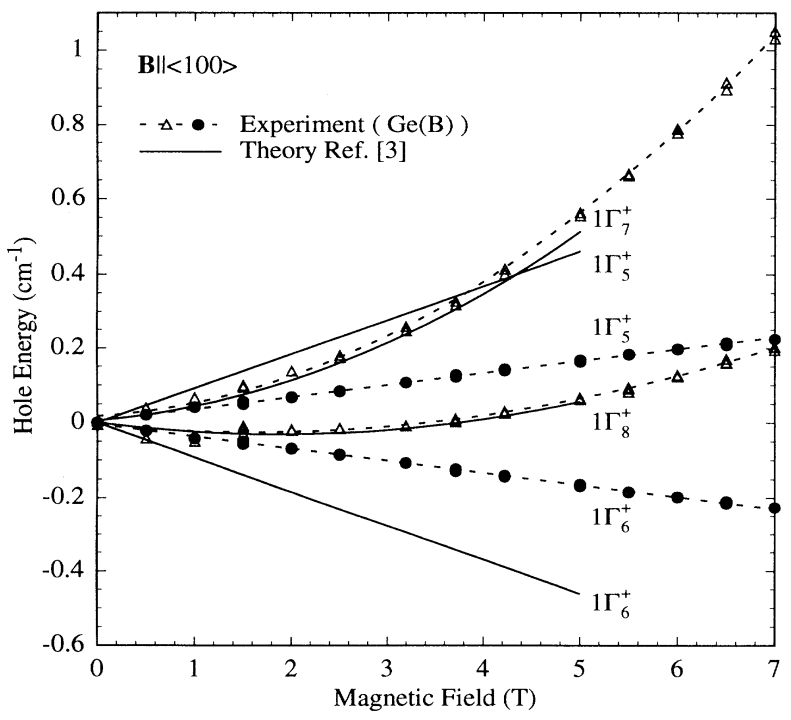

FIG. 3. Experimental Zeeman splitting of the ground state of $\mathrm{B}$ in Ge. The theoretical behavior is also shown, Ref. 3. there is excellent agreement between theory and experiment for the strongly field-dependent $\pm \frac{3}{2}$ states. The results of Ref. 4 give an ordering of the $\pm \frac{3}{2}$ states which is opposite both to that observed and that calculated in Ref. 3 , while the calculated splitting of the $\pm \frac{1}{2}$ states is $\sim 35 \%$ larger than that obtained in Ref. 3.

A comparison of the behavior of the ground states of $B$ and $\mathrm{Ga}$ is given in Fig. 4, where the splittings of the $\pm \frac{1}{2}$ and $\pm \frac{3}{2}$ states are shown for the ground states of both impurities; the dashed curves are for B while the full curves are for $\mathrm{Ga}$. These curves are least squares fits, those through the $\pm \frac{1}{2}$ data being linear fits while those through the $\pm \frac{3}{2}$ data are quadratic fits. For $B \gtrsim 1 \mathrm{~T}$, the ordering of the Zeeman states of the ground state of $\mathrm{Ga}$ is the same as that shown for B in Fig. 3. It is clear from Fig. 4 that the splitting of the $\pm \frac{3}{2}$ states for $\mathrm{Ga}$ is larger than the splitting of its $\pm \frac{1}{2}$ states at fields $\gtrsim 1 \mathrm{~T}$; this is also the case at very low fields as determined from the magnitudes of the linear terms of the fits to the splittings of these two pairs of states. This implies that, for $\mathrm{Ga}$, $1 \Gamma_{8}^{+}\left(\bar{C}_{4 h}\right)\left(+\frac{3}{2}\right)$ crosses $1 \Gamma_{6}^{+}\left(\bar{C}_{4 h}\right)\left(-\frac{1}{2}\right)$ at very low fields which is contrary to the results of Ref. 3, i.e., that the splitting of the $\pm \frac{3}{2}$ states is larger than that of the $\pm \frac{1}{2}$ states at low fields. For $\mathbf{B}$, the fits to the differences shown in Fig. 4 give linear terms for the $\pm \frac{3}{2}$ states which are smaller than those obtained for the $\pm \frac{1}{2}$ states. Thus for this impurity the arrangement of the Zeeman sublevels of the ground state at very low fields is the same as that given in Ref. 3 requiring $1 \Gamma_{7}^{+}\left(\bar{C}_{4 h}\right)\left(-\frac{3}{2}\right)$ to cross $1 \Gamma_{5}^{+}\left(\bar{C}_{4 h}\right)\left(+\frac{1}{2}\right)$ at a very much lower field than predicted.

The experimental data have been used to calculate $g$ factors. It is found, for example, that the $g$ factors $g_{1}^{\prime}$

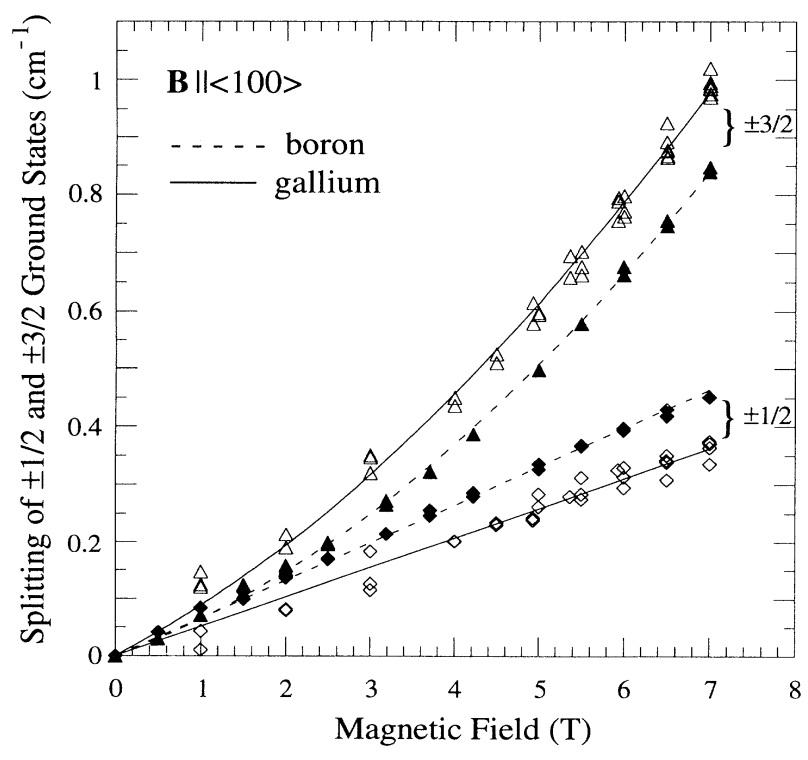

FIG. 4. Experimental Zeeman splitting of the $\pm \frac{1}{2}$ and $\pm \frac{3}{2}$ ground states of $\mathrm{B}$ and $\mathrm{Ga}$ in $\mathrm{Ge}$. The least squares fits through the $\pm \frac{1}{2}$ data are straight lines while those through the $\pm \frac{3}{2}$ data are quadratics. 
and $g_{2}^{\prime}$ of the ground state of B are $-0.164 \pm 0.001$ and $+0.091 \pm 0.001$, respectively, while those of $\mathrm{Ga}$ are $-0.132 \pm 0.002$ and $+0.084 \pm 0.002$, respectively. For $\mathrm{Ga}$, these are to be compared with $-0.16 \pm 0.08$ and $+0.08 \pm 0.04,{ }^{13}$ and $-0.19 \pm 0.02$ and $+0.05 \pm 0.01 .^{8}$ The theoretical values are -0.45 and $+0.22,{ }^{3}$ and -0.5904 and +0.2257 , respectively. ${ }^{4}$ In determining the present $g$ factors, the linear terms in the fits shown in Fig. 4 have been used. In Ref. 3, $g$ factors were determined by making quadratic fits to the numerical data generated by the computations, while in Ref. 4, they were calculated directly.

In conclusion, it is clear that the success of the Baldereschi-Lipari ${ }^{12}$ technique for calculating the eigenvalues of the unperturbed states of shallow acceptors in Ge has been equally successful when extended to the Zeeman behavior of these states. Also, the model used to de- scribe the acceptor ground state has had some success in that it predicts the linear splitting of the $\pm \frac{1}{2}$ states and the general behavior of the $\pm \frac{3}{2}$ states. It is also clear that the terms involved in the calculations of the magnitudes of these splittings need some modification to account for the smaller observed splitting of the $\pm \frac{1}{2}$ states and variations with chemical species. The other shallow impurities $\mathrm{Al}, \mathrm{In}, \mathrm{Tl}$, and $\mathrm{Zn}^{-}$in $\mathrm{Ge}$ are being studied as are the effects for other orientations of $\mathbf{B}$.

The authors wish to thank Professor W. O. G. Schmitt of the University of Würzberg for generously providing them with tables of calculated eigenvalues. They also acknowledge the support provided by the Australian Research Council and the University of Wollongong Board of Research and Postgraduate Studies to carry out the program.
${ }^{1}$ G. J. Takacs, R. E. M. Vickers, P. Fisher, and C. A. Freeth, Mater. Sci. Forum 117-118, 123 (1993).

${ }^{2}$ See, for example, A. K. Ramdas and S. Rodriguez, Rep. Prog. Phys. 44, 1297 (1981).

${ }^{3}$ J. Broeckx, Phys. Rev. B 43, 9643 (1991). Note that the behavior of the $2 \Gamma_{8}^{-}\left(\bar{O}_{h}\right)$ and $1 \Gamma_{8}^{+}\left(\bar{O}_{h}\right)$ states has been digitized from Figs. 1 and 3 for inclusion in Figs. 2 and 3 of the present work.

${ }^{4}$ W. O. G. Schmitt, E. Bangert, and G. Landwehr, J. Phys. Condens. Matter 3, 6789 (1991).

${ }^{5}$ H. P. Soepangkat and P. Fisher, Phys. Rev. B 8, 870 (1973).

${ }^{6}$ H. W. H. M. Jongbloets et al., J. Phys. C 13, 4769 (1980).

${ }^{7}$ J. Broeckx and P. Clauws, Solid State Commun. 28, 355 (1978); J. Broeckx et al., J. Phys. C 12, 4061 (1979).

${ }^{8}$ K. J. Duff et al., in Proceedings of the 19th International Conference on the Physics of Semiconductors, edited by W.
Zawadzki (Institute of Physics, Polish Academy of Science, 1988), p. 1273.

${ }^{9}$ G. Jungwirt and W. Prettl, Int. J. Infrared Millimeter Waves 10, 1033 (1989).

${ }^{10}$ P. J. Lin-Chung and R. F. Wallis, J. Phys. Chem. Solids 30, 1453 (1969).

${ }^{11}$ A. K. Bhattercharjee and S. Rodriguez, Phys. Rev. B 6, 3836 (1972).

12 A. Baldereschi and N. O. Lipari, Phys. Rev. B 8, 2697 (1973); 9, 1525 (1974). See also Proceedings of the 13th International Conference on the Physics of Semiconductors, edited by F. G. Fumi (Tipografia Marves, Rome, 1976), p. 595 and N. O. Lipari and A. Baldereschi, Solid State Commun. 25, 665 (1978).

${ }^{13}$ H. Tokumoto and T. Ishiguro, Phys. Rev. B 15, 2099 (1977). 